\title{
Consequences of visitor restriction policies in the intensive care unit during the COVID-19 pandemic
}

\author{
Kimia Honarmand, MD, MSc (iD) Sangeeta Mehta, MD
}

Received: 7 May 2021/Revised: 7 May 2021/ Accepted: 8 June 2021 / Published online: 1 July 2021

(C) Canadian Anesthesiologists' Society 2021

Being in the intensive care unit (ICU) may be an impersonal, overwhelming, and at times dehumanizing experience for patients. Critically ill patients experience physical and psychological distress and require interdisciplinary care to address their complex needs and reduce suffering. Family members are increasingly recognized as essential partners in addressing the complex cognitive, emotional, social, and practical needs of ICU patients. This recognition has led to a trend towards gradual liberalization of ICU visitation policies in Canada and around the world. ${ }^{1,2}$ "Open visitation" in the ICU expands family presence and their role to mitigate the psychological impact of the ICU environment and critical illness on patients, reduce distress among family members, and foster communication and collaboration between families and the care team.

Visitor restrictions during the COVID-19 pandemic have led to an abrupt halt in successful initiatives to expand family presence and involvement in the care of ICU patients. Faced with a rapidly spreading disease, limited knowledge about transmission, and possible shortages in resources (e.g., personal protective equipment [PPE]), hospitals have expeditiously implemented revised visitation policies that have drastically restricted or even eliminated family presence. Given variations in community spread and resource availability across jurisdictions,

\footnotetext{
K. Honarmand, MD, MSc ( $\varangle)$

Department of Medicine, Division of Critical Care, Western

University, London, ON, Canada

e-mail: kimia.honarmand@medportal.ca

S. Mehta, MD

Department of Medicine, Sinai Health and Interdepartmental Division of Critical Care Medicine, University of Toronto, Toronto, ON, Canada
}

developing and implementing visitor restriction policies fell to individual healthcare facilities, leading to variability and confusion about visitation rules even in hospitals within the same jurisdiction among patients, families, and frontline clinicians.

In this issue of CJA, Fiest et al. report the findings of their environmental scan which describes the extent, variations, and fluctuations of Canadian adult ICU visitation restrictions before (preCOVID) and at two timepoints (midCOVID and lateCOVID) during the first wave of the COVID-19 pandemic in Canada. ${ }^{3}$ Based on 257 visitation policy documents from 230 Canadian hospitals/ ICUs, they report that preCOVID, over twothirds of ICUs permitted “open" (24-hr) visitation. Unsurprisingly, none of the hospitals retained open visitation during the pandemic, with the majority adopting a "no visitor" policy with pre-specified exceptions (midCOVID: 86\%; lateCOVID: 76\%). All policy documents described the rationale for visitor restrictions, most commonly citing "protecting the public and hospital occupants" and "preserving resources such as PPE".

While most policies listed exceptions to visitation restrictions, most commonly for patients at the end of life, only about one in ten defined the patients that would meet the criteria. When criteria were listed, definitions were variable, ranging from predictive scales (e.g., higher Palliative Performance Score) to clinical judgement that death is imminent. Some ICUs had more liberal visitation policies based on the patient's clinical status (e.g., longer length of ICU stay). Interestingly, less than one-third of midCOVID visitation policies made exceptions to visitor restrictions for patients requiring cognitive or physical assistance, in contrast to $39 \%$ of lateCOVID visitation policies, perhaps reflecting increased appreciation of the 
adverse consequences of isolation on patients and their families. For patients with suspected or confirmed COVID19 , fewer than $25 \%$ of hospitals permitted any visitation and only $7 \%$ allowed visitation on a case-by-case basis (e.g., at the end of life). About half of the policy documents offered alternative modalities to connect patients with families (e.g., video calls).

While drastic visitor restrictions were likely unavoidable during the early phases of the pandemic, they contributed to patient isolation, feelings of helplessness, and anxiety among family members, and moral distress among clinicians. Family presence provides comfort, assurance, and a sense of familiarity to patients during the overwhelming experience of critical illness. Consequences of reduced family presence include higher incidence and severity of delirium, ${ }^{4,5}$ higher anxiety levels, ${ }^{6,7}$ reduced patient rest, ${ }^{8,9}$ and more cardiovascular complications. ${ }^{7}$ Visitor restrictions isolate patients and deprive them of the benefits of family caregivers.

Family caregivers experience psychological sequelae after critical illness of a loved one. ${ }^{10,11}$ In studies before the COVID-19 pandemic, liberal visitation policies were associated with lower anxiety, ${ }^{12}$ less exhaustion, ${ }^{9}$ and higher satisfaction ${ }^{12,13}$ among families of ICU patients. In our experience, family members who are restricted from visiting experience profound distress, uncertainty, helplessness, and at times mistrust of the care team. Physical presence in the ICU may also help families better comprehend a patient's clinical status (and possibly suffering) and facilitate more timely dialogue between families and the care team. Indeed, one study reported that restricting visitation delayed conversations about goals of care during the COVID-19 pandemic. $^{14}$

Among the most tragic consequences of visitor restrictions is inability of the family to be present when a patient is dying. Although most policies offer some provisions for dying patients, they limit the number of visitors, and patients or family members with suspected or confirmed COVID-19 are disallowed. Prohibiting family presence at the end of a patient's life deprives patients of solace and connectedness in the last moments of life and may lead to complicated grief and prolonged psychological trauma among families.

The COVID-19 pandemic has had a heavy toll on frontline workers, ${ }^{15}$ which has been compounded by restricted visitation. In our survey of 1,875 hospital workers in Ontario, $80 \%$ of respondents reported concerns about the adverse impact of visitor restrictions on the well-being of their patients (unpublished data). Frontline clinicians are often the face of hospital policies despite having limited or no role in their development. Being the enforcers of visitor restrictions that may have adverse effects on patients and families can exacerbate moral distress and increase psychological burden. This is particularly the case at the end of life, when frontline clinicians have taken the place of families at the bedside of patients that are dying alone and have remotely consoled grieving family members.

As we continue to battle the COVID-19 pandemic, it is prudent to re-conceptualize the balance between patientand family-centred care with our obligation to reduce transmission. ${ }^{16}$ Although policies must be adapted to local circumstances and resources, several common principals pertain to the development and enforcement of visitation policies during public health crises. Visitation policies should be developed with direct engagement of representatives from groups who will be directly impacted. Input from patient and family partners and frontline clinicians with various levels of experience will ensure that policies are humane, equitable, trustworthy, and practical.

Visitation policies should be as liberal as local circumstances allow, to minimize the adverse impact on patients, families, and clinicians. Where restrictions are deemed necessary, the specific restrictions and the rationale should be communicated to those who are impacted. This information should be easily accessible in public-facing hospital websites and clearly visible posters in the hospital, in language that is adapted to various communication styles and education levels. Alternative modalities of connecting patients and families (e.g., videoconferencing) should be offered to mitigate the adverse consequences of visitor restriction policies.

Exceptions to visitor restriction should be carefully considered, particularly for patients at the end of life and for particularly vulnerable groups, including those with acute or chronic cognitive impairment, specific communication needs (e.g., hearing impairment), and language differences. Any exceptions to each policy item should be clearly defined to avoid confusion or inequitable enforcement.

A transparent appeals process should be made available, and efforts made to ensure the process is easily accessible to patients and families. Given the racial and socioeconomic disparities in COVID-19 transmission and outcomes, broad restrictions in visitation may disproportionally impact racialized and under-privileged communities and strategies to mitigate these disparities are encouraged (e.g., lending hospital-owned devices to families who lack access to communication devices).

Delegating policy enforcement to bedside clinicians may impair the relationship between clinicians and patients/families and contribute to clinician distress. Communicating and enforcing visitor restriction policies should be done by those unconnected to the patient's care. To optimize family support in a busy pandemic ICU, ICUs 
could establish family liaison/ communication teams, consisting of clinicians and trainees from other specialties who can regularly liaise with each patient's family, facilitate telephone or video calls with patients, and arrange for and guide families during in-person visits where permitted (i.e., meet family members when they arrive, reinforce visitation policies such as handwashing, guide and observe PPE donning and doffing, and offer emotional support). Such family liaison/communication teams would likely reduce patient/family distress by facilitating more frequent patient-family engagement, while preserving the important role of ICU clinicians in communicating medical information with families.

The COVID-19 pandemic has compelled our healthcare system to face the insurmountable challenge of balancing our long-standing mission to promote compassionate patient- and family-centred care with our obligation to protect patients, family members, healthcare staff, and the community. The collective pursuit towards navigating these conflicting commitments is likely to generate innovations that will transform patient- and familycentred care in the ICU.

\section{Conséquences des politiques de restriction des visites à l'unité de soins intensifs pendant la pandémie de COVID-19}

Un séjour à l'unité de soins intensifs (USI) peut être une expérience impersonnelle, accablante, voire déshumanisante pour les patients. Les patients en état critique éprouvent une détresse physique et psychologique et demandent des soins interdisciplinaires pour répondre à leurs besoins complexes et réduire leur souffrance. Il est de plus en plus reconnu que les membres de la famille sont des partenaires essentiels pour répondre aux besoins cognitifs, émotionnels, sociaux et pratiques complexes des patients pris en charge aux soins intensifs. Cette reconnaissance a entraîné dans son sillon une libéralisation progressive des politiques de visites aux soins intensifs au Canada et ailleurs dans le monde. ${ }^{1,2}$ Les « visites libres » à l'USI étendent la présence des membres de la famille et leur rôle afin d'atténuer l'impact psychologique de l'environnement des soins intensifs et des maladies graves sur les patients, de réduire la détresse chez les membres de la famille, et de favoriser la communication et la collaboration entre les familles et l'équipe soignante.
Les restrictions imposées aux visiteurs pendant la pandémie de COVID-19 ont entraîné un arrêt soudain d'initiatives efficaces visant à augmenter la présence des familles et leur participation aux soins des patients à l'USI. Confrontés à une maladie à propagation rapide, à des connaissances limitées sur la transmission et à la menace de pénuries de ressources (p. ex., équipements de protection individuelle [EPI]), les hôpitaux ont rapidement mis en œuvre des politiques révisées de visites qui ont radicalement restreint, voire éliminé la présence familiale. Compte tenu des variations dans la transmission communautaire et la disponibilité des ressources entre les régions, l'élaboration et la mise en œuvre des politiques de restriction des visites ont incombé directement aux établissements de soins de santé individuels, ce qui a entraîné une variabilité et de la confusion parmi les patients, les familles et les cliniciens de première ligne concernant les règles de visite et ce, même dans des hôpitaux d'une même juridiction.

Dans ce numéro du Journal, Fiest et coll. présentent les résultats de leur étude de milieu qui décrit l'ampleur, les variations et les fluctuations des restrictions relatives aux visites aux unités de soins intensifs pour adultes au Canada avant (pré-COVID) et à deux points de temps (mid-COVID et COVID-tardif) au cours de la première vague de la pandémie de COVID-19 au Canada. ${ }^{3}$ Sur la base de 257 documents décrivant les politiques de visite de 230 hôpitaux/USI canadiens, les auteurs rapportent qu'avant la COVID, plus des deux tiers des USI autorisaient les visites « libres» (24 heures sur 24). Sans surprise, aucun hôpital n'a maintenu les visites libres pendant la pandémie, la majorité adoptant une politique de « visites interdites » hormis pour des exceptions prédéterminées (mid-COVID : $86 \%$; COVID-tardif : $76 \%$ ). Tous les documents de politiques décrivaient la raison d'être des restrictions imposées aux visiteurs, citant le plus souvent « la protection du public et des occupants de l'hôpital » et « la préservation des ressources telles que les EPI ».

Alors que la plupart des politiques énuméraient des exceptions aux restrictions de visite, le plus souvent pour les patients en fin de vie, seulement une sur dix environ définissait quels patients répondaient à ces critères. Lorsque les critères étaient énumérés, les définitions variaient, allant d'échelles prédictives (p. ex., score plus élevé sur l'Échelle de performance pour soins palliatifs) au jugement clinique que le décès était imminent. Certaines USI avaient des politiques de visites plus libérales fondées sur l'état clinique du patient (p. ex., une plus longue durée de séjour aux soins intensifs). Fait intéressant, moins du tiers des politiques de visites mid-COVID ont fait des exceptions aux restrictions imposées aux visiteurs pour les patients nécessitant une assistance cognitive ou physique, contrairement à $39 \%$ des politiques de visites en période 
COVID-tardif, ce qui reflète peut-être une meilleure compréhension des conséquences néfastes de l'isolement sur les patients et leurs familles. Pour les patients avec suspicion ou confirmation de COVID-19, moins de $25 \%$ des hôpitaux ont autorisé des visites et seulement $7 \%$ ont autorisé des visites au cas par cas (p. ex., en fin de vie). Environ la moitié des documents de politiques proposaient d'autres modalités pour que les patients gardent contact avec leur famille (p. ex., appels vidéo).

Bien que des restrictions drastiques imposées aux visiteurs aient probablement été inévitables au cours des premières phases de la pandémie, elles ont contribué à l'isolement des patients, au sentiment d'impuissance et d'anxiété des membres de la famille, ainsi qu'à la détresse morale des cliniciens. La présence familiale procure réconfort, assurance et sentiment de familiarité aux patients au cours de l'expérience accablante d'une maladie grave. Parmi les conséquences d'une présence familiale réduite, citons une incidence et une gravité plus élevées de delirium, ${ }^{4,5}$ des niveaux d'anxiété plus élevés, ${ }^{6,7}$ une réduction du repos des patients, ${ }^{8,9}$ et une augmentation des complications cardiovasculaires. ${ }^{7}$ Les restrictions des visites isolent les patients et les privent des bienfaits des aidants naturels.

Les aidants naturels éprouvent des séquelles psychologiques après la maladie grave d'un être cher. ${ }^{10,11}$ Dans les études menées avant la pandémie de COVID-19, les politiques de visites libérales étaient associées à une anxiété plus faible, ${ }^{12}$ un épuisement moindre, ${ }^{9}$ et une plus grande satisfaction ${ }^{12,13}$ parmi les familles de patients aux soins intensifs. D'après notre expérience, les membres de la famille qui ne peuvent pas rendre visite à leurs proches éprouvent une profonde détresse, de l'incertitude, de l'impuissance, voire de la méfiance à l'égard de l'équipe soignante. Leur présence physique aux soins intensifs pourrait également aider les familles à mieux comprendre l'état clinique d'un patient (et peut-être sa souffrance) et faciliter un dialogue plus opportun entre les familles et l'équipe soignante. En effet, une étude a indiqué que la restriction des visites avait retardé les conversations sur les objectifs de soins pendant la pandémie de COVID-19. ${ }^{14}$

Parmi les conséquences les plus tragiques des restrictions des visites, citons l'incapacité des familles d'être présentes lors du décès d'un patient. Bien que la plupart des politiques incluent certaines dispositions pour les patients mourants, elles limitent toutefois le nombre de visiteurs, et les patients ou les membres de la famille soupçonnés ou confirmés COVID-19 ne peuvent en bénéficier. Interdire la présence familiale d'un patient en fin de vie prive les patients de réconfort et de connexion dans les derniers moments de leur vie et peut entraîner un deuil compliqué et un traumatisme psychologique prolongé pour les familles.
La pandémie de COVID-19 a eu de lourdes séquelles pour les travailleurs de première ligne, ${ }^{15}$ d'autant plus aggravées par les restrictions des visites. Dans notre sondage mené auprès de 1875 travailleurs hospitaliers en Ontario, $80 \%$ des répondants se sont dits préoccupés par l'incidence néfaste des restrictions imposées aux visiteurs sur le bien-être de leurs patients (données non publiées). Les cliniciens de première ligne sont souvent le visage des politiques hospitalières et ce, malgré leur rôle limité ou nul dans leur mise au point. Le fait d'être l'exécuteur des restrictions imposées aux visiteurs qui pourraient avoir des effets néfastes sur les patients et leurs familles peut exacerber la détresse morale et augmenter le fardeau psychologique de ces travailleurs. C'est particulièrement le cas en fin de vie, lorsque les cliniciens de première ligne ont pris la place de familles au chevet de patients mourant seuls et qu'ils consolent les membres de leur famille en deuil à distance.

Alors que nous continuons de lutter contre la pandémie de COVID-19, il serait judicieux de repenser l'équilibre entre les soins centrés sur le patient et sur la famille et notre devoir de réduire la transmission. ${ }^{16}$ Bien que les politiques doivent être adaptées aux circonstances et aux ressources locales, plusieurs principes communs touchent à l'élaboration et à l'application des politiques de visites pendant les crises de santé publique. Les politiques de visite devraient être élaborées avec l'implication directe de représentants des groupes qui seront directement touchés. Les contributions des patients, des partenaires familiaux et des cliniciens de première ligne ayant divers niveaux d'expérience feront en sorte que les politiques seront humaines, équitables, dignes de confiance et applicables.

Afin de minimiser l'impact négatif sur les patients, les familles et les cliniciens, les politiques de visite devraient être aussi libérales que les circonstances locales le permettent. Lorsque des restrictions sont jugées nécessaires, les restrictions spécifiques et leur justification devraient être communiquées aux personnes touchées. Ces informations devraient être facilement accessibles sur les sites internet publics des hôpitaux et par le biais d'affiches clairement visibles à l'hôpital, dans un langage adapté aux différents styles de communication et niveaux d'éducation. D'autres modalités de mise en relation des patients et des familles (p. ex., vidéoconférence) devraient être proposées pour atténuer les conséquences néfastes des politiques de restriction s'appliquant aux visiteurs.

Les exceptions aux restrictions des visites devraient être soigneusement examinées, en particulier pour les patients en fin de vie et pour les groupes particulièrement vulnérables, notamment pour les personnes qui souffrent d'une déficience cognitive aiguë ou chronique, ont des besoins spécifiques en matière de communication (p. ex., déficience auditive) ou présentent des différences 
linguistiques. Toute exception à des éléments d'une politique doit être clairement définie afin d'éviter toute confusion ou toute application inéquitable.

Un processus de recours transparent devrait être mis à disposition et des efforts devraient être déployés pour s'assurer que le processus est facilement accessible aux patients et aux familles. Compte tenu des disparités raciales et socioéconomiques dans la transmission et les devenirs de la COVID-19, les restrictions importantes dans les visites pourraient avoir un impact disproportionné sur les communautés racialisées et défavorisées; c'est pourquoi des stratégies visant à atténuer ces disparités sont encouragées (p. ex., prêt d'appareils appartenant à l'hôpital à des familles qui n'ont pas accès aux dispositifs de communication).

Le fait de déléguer l'application des politiques aux cliniciens de première ligne peut nuire à la relation entre les cliniciens et les patients/familles et contribuer à la détresse des cliniciens. La communication et l'application des politiques de restriction des visites devraient être sous la responsabilité de personnes n'ayant aucun lien avec les soins du patient. Afin d'optimiser le soutien familial dans une unité de soins intensifs occupée en temps de pandémie, les USI pourraient mettre sur pied des équipes de liaison et de communication avec les familles, composées de cliniciens et de stagiaires d'autres spécialités qui pourraient assurer une liaison régulière avec la famille de chaque patient, faciliter les appels téléphoniques ou vidéo avec les patients, et organiser et guider les familles lors de visites en personne lorsque cela est permis (c.-à-d. rencontrer les membres de la famille à leur arrivée, renforcer les politiques de visite comme le lavage des mains, guider et observer l'enfilage et le retrait des EPI, et offrir un soutien émotionnel). De telles équipes de liaison et de communication avec la famille réduiraient probablement la détresse des patients et des familles en facilitant des interactions plus fréquentes entre le patient et sa famille, tout en préservant le rôle important des cliniciens des soins intensifs dans la communication de renseignements médicaux avec les familles.

La pandémie de COVID-19 a forcé notre système de soins de santé à relever le défi insurmontable d'assurer un équilibre entre notre mission de longue date de promouvoir des soins centrés sur le patient et la famille empreints de compassion, et notre obligation de protéger les patients, les membres de leurs familles, le personnel de santé et la communauté. La poursuite collective visant à trouver une voie dans ces engagements contradictoires est susceptible de générer des innovations qui transformeront les soins centrés sur le patient et la famille aux soins intensifs.

Disclosures None.
Funding statement None.

Editorial responsibility This submission was handled by Dr. Stephan K.W. Schwarz, Editor-in-Chief, Canadian Journal of Anesthesia.

Déclaration Aucune.

Déclaration de financement Aucune.

Responsabilité éditoriale Ce manuscrit a été traité par Dr Stephan K.W. Schwarz, rédacteur en chef, Journal canadien d'anesthésie.

\section{References}

1. Cappellini E, Bambi S, Lucchini A, Milanesio E. Open intensive care units: a global challenge for patients, relatives, and critical care teams. Dimens Crit Care Nurs 2014; 33(4): 181-93.

2. Nassar Junior AP, Besen B, Robinson CC, Falavigna M, Teixeira $C$, Rosa $R G$. Flexible Versus Restrictive Visiting Policies in ICUs: A Systematic Review and Meta-Analysis. Crit Care Med 2018; 46(7): 1175-80.

3. Fiest KM, Krewulak KD, Hiploylee $C$, et al. An environmental scan of visitation policies in Canadian intensive care units during the first wave of the COVID-19 pandemic. Can J Anesth 2021; this issue. https://doi.org/10.1007/s12630-021-02049-4

4. Rosa RG, Tonietto TF, da Silva DB, et al. Effectiveness and Safety of an Extended ICU Visitation Model for Delirium Prevention: A Before and After Study. Crit Care Med 2017; 45(10): 1660-7.

5. Eghbali-Babadi M, Shokrollahi N, Mehrabi T. Effect of FamilyPatient Communication on the Incidence of Delirium in Hospitalized Patients in Cardiovascular Surgery ICU. Iran J Nurs Midwifery Res 2017; 22(4): 327-31.

6. Fumagalli S, Calvani S, Gironi E, et al. An unrestricted visitation policy reduces patients' and relatives' stress levels in intensive care units. European Heart Journal 2013; 34(suppl_1).

7. Fumagalli S, Boncinelli L, Lo Nostro A, et al. Reduced cardiocirculatory complications with unrestrictive visiting policy in an intensive care unit: results from a pilot, randomized trial. Circulation 2006; 113(7): 946-52.

8. Roland P, Russell J, Richards KC, Sullivan SC. Visitation in critical care: processes and outcomes of a performance improvement initiative. J Nurs Care Qual 2001; 15(2): 18-26.

9. Petterson $M$. Process helped gain acceptance for open visitation hours. Crit Care Nurse 2005; 25(1): 72, 0-1.

10. Cameron JI, Chu LM, Matte A, et al. One-year outcomes in caregivers of critically ill patients. NEJM 2016; 374: 1831-41.

11. Herridge MS, Moss M, Hough CL, et al. Recovery and outcomes after the acute respiratory distress syndrome (ARDS) in patients and their family caregivers. Intensive Care Med 2016; 42(5): 725-38.

12. Garrouste-Orgeas M, Philippart F, Timsit JF, et al. Perceptions of a 24-hour visiting policy in the intensive care unit. Crit Care Med 2008; 36(1): 30-5.

13. Goldfarb MJ, Bibas L, Bartlett V, Jones $H$, Khan N. Outcomes of Patient- and Family-Centered Care Interventions in the ICU: A Systematic Review and Meta-Analysis. Crit Care Med 2017; 45(10): 1751-61.

14. Azad TD, Al-Kawaz MN, Turnbull AE, Rivera-Lara $L$. Coronavirus Disease 2019 Policy Restricting Family Presence 
May Have Delayed End-of-Life Decisions for Critically Ill Patients. Crit Care Med 2021.

15. Mehta S, Machado F, Kwizera A, et al. COVID-19: a heavy toll on health-care workers. Lancet Respir Med 2021; 9(3): 226-8.

16. Munshi L, Evans G, Razak $F$. The case for relaxing no-visitor policies in hospitals during the ongoing COVID-19 pandemic. CMAJ 2021; 193(4): E135-7.
Publisher's Note Springer Nature remains neutral with regard to jurisdictional claims in published maps and institutional affiliations. 